\title{
Reflets
}

Revue ontaroise d'intervention sociale et communautaire

\section{Corps, santé, apparence et vieillissement dans les énoncés de femmes francophones en Ontario}

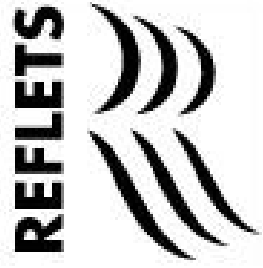

\section{Natalie Beausoleil}

Volume 4, numéro 1, printemps 1998

Intervention en contextes minoritaires

URI : https://id.erudit.org/iderudit/026197ar

DOI : https://doi.org/10.7202/026197ar

Aller au sommaire du numéro

Éditeur(s)

Reflets : Revue ontaroise d'intervention sociale et communautaire

ISSN

1203-4576 (imprimé)

1712-8498 (numérique)

Découvrir la revue

Citer cet article

Beausoleil, N. (1998). Corps, santé, apparence et vieillissement dans les énoncés de femmes francophones en Ontario. Reflets, 4(1), 53-74.

https://doi.org/10.7202/026197ar
Résumé de l'article

Cet article examine le rapport au corps et la représentation de soi de quelques femmes francophones en Ontario, à partir d'entretiens faits à Ottawa, Sudbury et Toronto. La première partie du texte montre comment les femmes francophones minoritaires partagent avec d'autres femmes de la société occidentale contemporaine des préoccupations de vieillissement, de santé et d'apparence. La deuxième partie du texte propose quelques jalons pour l'étude des spécificitésdu vécu corporel de femmes francophones minoritaires. Ce texte souligne l'importance decomprendre les complexités du vécu des femmes et leur lucidité face aux obligations corporellesqui façonnent leur identité et leur santé.
Tous droits réservés $@$ Reflets : Revue ontaroise d'intervention sociale et communautaire, 1998
Ce document est protégé par la loi sur le droit d'auteur. L'utilisation des services d’Érudit (y compris la reproduction) est assujettie à sa politique d'utilisation que vous pouvez consulter en ligne.

https://apropos.erudit.org/fr/usagers/politique-dutilisation/ 


\section{Corps, santé, apparence et vieillissement dans les énoncés de femmes francophones en Ontario ${ }^{1}$}

Cet article examine le rapport au corps et la représentation de soi de quelques femmes francophones en Ontario, à partir d'entretiens faits à Ottawa, Sudbury et Toronto. La première partie du texte montre comment les femmes francophones minoritaires partagent avec d'autres femmes de la société occidentale contemporaine des préoccupations de vieillissement, de santé et d'apparence. La deuxième partie du texte propose quelques jalons pour l'étude des spécificités du vécu corporel de femmes francophones minoritaires. Ce texte souligne l'importance de comprendre les complexités du vécu des femmes et leur lucidité face aux obligations corporelles qui façonnent leur identité et leur santé.

\section{Natalie Beausoleil}

Professeure, Département de sociologie, Université d'Ottawa

\section{Introduction : l'étude de la corporéité et les champs de la santé et du vieillissement}

Cet article s'inscrit dans une investigation plus large de la production des identités des femmes francophones en Ontario par et à travers le corps et l'apparence ${ }^{2}$. Ma recherche examine les questions suivantes : comment est-ce que la culture s'inscrit sur les corps des francophones en Ontario? Est-ce que les femmes francophones en Ontario produisent leurs corps comme signes distinctifs de la majorité anglo-saxonne, et si oui, comment? Plus 
spécifiquement, j'ai commencé à étudier certains processus de production d'identité et de minorisation chez les FrancoOntariennes. Les travaux récents dans le champ des francophonies minoritaires suggèrent l'éclatement et les fluctuations de la francophonie hors Québec (Cardinal 1994). Comment les femmes appartenant à une diversité de classes sociales, d'âges, de groupes ethniques, définissent-elles et vivent-elles leur francophonie?

Dans cet article, je décris et j'analyse comment quelques femmes lient apparence, santé et vieillissement dans leurs énoncés sur le corps. Afin d'examiner de façon empirique comment des femmes vivent leur corps, j'ai choisi d'utiliser une approche qualitative (Campbell et Manicom 1995; Deslauriers 1991; DeVault 1990; Emerson 1983; Huberman et Miles 1991; Mishler 1986; Roberts 1981; Smith 1987). Plus spécifiquement, j’ai eu recours à des entretiens individuels, de type semi-structuré ${ }^{3}$. Ce type de méthodologie permet d'explorer les pratiques des femmes à partir de leurs préoccupations. ${ }^{4}$ Ces entretiens permettent aux femmes d'élaborer sur ce qu'elles trouvent important et significatif dans le domaine de recherche. Dans cet article, j'utilise quelques extraits d'entretiens parmi les trente (30) que j'ai effectués avec des femmes francophones d'Ottawa, de Sudbury et de Toronto ${ }^{5}$. Les femmes rencontrées sont d'âges très divers et chacune a ses préoccupations particulières. Ainsi les femmes de quarante ans et plus ont parlé des dimensions de vieillissement, de santé et d'apparence dans leurs vies. Les entretiens avec ces femmes sont analysés dans la première partie de ce texte. D'autre part, des femmes de tous les âges ont exprimé leurs préoccupations quant à l'ethnicisation du corps. La deuxième partie de l'article reflète cette diversité dans l'âge des femmes rencontrées; le corpus des entretiens y est donc plus étendu. Les grands thèmes suivants sont ressortis des entretiens: la perception du corps, l'identité francophone, les pratiques d'apparence quotidienne (maquillage, vêtements, soin des cheveux, bijoux, etc.), la santé,le vieillissement, les relations aux autres et les commentaires sur le corps dans les interactions. Chaque entretien a été analysé individuellement (analyse verticale) et les thèmes relevés dans les entretiens ont ensuite été liés les uns aux autres (analyse horizontale). Notez 
"Les entretiens révèlent que les femmes francophones minoritaires en Ontario partagent plusieurs pratiques et préoccupations corporelles avec l'ensemble des femmes de la société contemporaine occidentale. D'autre part, certaines femmes suggèrent que le corps est vécu de façon «différente» de la majorité pour les femmes francophones minoritaires. Parce que les femmes francophones minoritaires vivent à la fois la «similarité» et la "différence», il apparait crucial d'analyser ces deux dimensions." que tous les noms utilisés ici sont des pseudonymes afin de protéger l'anonymat des femmes rencontrées. ${ }^{6}$

L'intérêt de cette recherche consiste à explorer comment la corporéité se vit pour les femmes en ce qui touche à la santé et au vieillissement. Cette recherche part donc du postulat qu' il est crucial de comprendre l'expérience des femmes dans les domaines de la santé et du vieillissement afin de pouvoir intervenir adéquatement auprès des femmes de diverses communautés. Dans une approche sociologique et anthropologique plutôt qu'épidémiologique, cette recherche examine les contextes sociaux et culturels qui façonnent comment les femmes vivent la santé et la vieillissement. L'approche sociologique et anthropologique permet d'étudier la construction sociale des questions de santé dans toute sa complexité (Corin et al 1990). Cette recherche privilégie donc comme point de départ le sens que donnent les femmes aux problématiques étudiées.

Les entretiens révèlent que les femmes francophones minoritaires en Ontario partagent plusieurs pratiques et préoccupations corporelles avec l'ensemble des femmes de la société contemporaine occidentale. D'autre part, certaines femmes suggèrent que le corps est vécu de façon «différente» de la majorité pour les femmes francophones minoritaires. Parce que les femmes francophones minoritaires vivent à la fois la «similarité» et la «différence», il apparait crucial d'analyser ces deux dimensions. Dans cette perspective, la première partie porte sur quelques aspects du rapport au corps que les femmes francophones minoritaires vivent en commun avec d'autres femmes de la société occidentale contemporaine, en l'occurrence l'articulation santé-apparencevieillissement. Dans la deuxième partie du texte, je propose quelques pistes d'investigation pour commencer à comprendre comment la minorisation des femmes francophones en Ontario s'inscrit sur leur corps. J'explore ici uniquement l'ethnicisation du corps telle que vécue par des femmes francophones minoritaires de race blanche. Dans un article en préparation,j'analyse les liens entre la diversité raciale et l'ethnicisation du corps parmi les femmes francophones minoritaires. 


\section{Santé, veillissement et apparence dans les énoncés des femmes rencontrées}

\section{La recherche du bien-être}

En quoi consiste la santé pour les femmes rencontrées? Où commence et où se termine le domaine de la santé dans leurs discours sur le corps et les apparences? Pour certaines, la question

"Les femmes plus âgées en particulier ont immédiatement soulevé les questions de la santé lorsque je leur ai demandé de me parler du corps et des apparences.» du corps est premièrement et surtout une question de santé. Les femmes plus âgées en particulier ont immédiatement soulevé les questions de la santé lorsque je leur ai demandé de me parler du corps et des apparences. Ce résultat n'est pas surprenant. Toutes les femmes sont préoccupées par la santé, mais en vieillissant, cet aspect du rapport au corps prend de plus en plus d'importance. Le cycle de vie, ou l' âge des femmes, marque donc de façon significative le rapport entre corps et santé. Que signifie avoir une bonne santé pour les femmes rencontrées? Une bonne santé consiste à atteindre (ou maintenir) le bien-être en général. Les composantes du bien-être qui ressortent des énoncés des femmes sont les suivantes: une alimentation saine, de l'exercice régulier et le port de bonne chaussures. Ces éléments lient le confort au bien-être global qui caratérise la santé. Les femmes rencontrées désirent atteindre un certain équilibre et vivre sans excès. Dans cette perspective, elles élaborent des stratégies quotidiennes variées.

Les femmes âgées ne forment pas un groupe homogène (Ladouceur, 1996). Leurs différentes situations sociales et les configurations personnelles de leur vies façonnent leur rapport au corps. Les entretiens effectués démontrent que le vieillissement se vit de diverses façons pour des femmes dans des contextes sociaux différents. Si par exemple une femme de classe moyennesupérieure en bonne santé vit de façon agréable la retraite et le vieillissement, les femmes qui vivent dans la pauvreté envisagent le vieillissement avec appréhension. Pour certaines, vieillir fait peur largement à cause des problèmes de santé et des problèmes financiers qui y sont associés. Celles qui sont déjà malades en 
particulier sont appréhensives face au vieillissement en terme de détérioration de leur santé, déjà précaire.

Les dimensions esthétiques de la santé et du vieillissement constituent une préoccupation importante pour plusieurs femmes rencontrées, entre autres parmi les femmes dans la quarantaine et la cinquantaine. L'apparence corporelle est un facteur non négligeable marquant le processus de vieillissement. Dans les sections suivantes, j'analyse comment l'apparence fait partie des préoccupations de santé et de veillissement des femmes rencontrées.

\section{Poids et changements : la gêne et l'apprivoisement du corps}

«Plusieurs femmes rencontrées dénoncent les privations et efforts exigés des femmes, telles les privations requises en suivant des régimes, privations qui se poursuivent jusque dans la vieillesse. Mais même ces femmes qui sont critiques des normes sociales expriment néanmoins la peur des changements du corps qui annoncent le vieillissement.»
Préoccupations de santé et esthétique sont inextricablement liées dans la question du poids du corps. La pratique des régimes affecte l'entretien du corps et la santé de même qu'elle renvoie à des critères esthétiques. L'obligation de la minceur frappe particulièrement les femmes (Bordo 1993; Chernin 1981; King 1994; Millman 1980; Szekeley 1988). Cette obligation a un impact psychologique non négligeable pour l'ensemble des femmes dans la société occidentale contemporaine. Elle a aussi inextricablement des conséquences graves sur leur santé. Les femmes qui suivent des régimes et/ou cessent presque complètement de manger font des torts parfois irréparables à leur santé. Cette minceur (ou maigreur) est inatteignable pour presque toutes. ${ }^{7}$ Plusieurs femmes rencontrées dénoncent les privations et efforts exigés des femmes, telles les privations requises en suivant des régimes, privations qui se poursuivent jusque dans la vieillesse. Mais même ces femmes qui sont critiques des normes sociales expriment néanmoins la peur des changements du corps qui annoncent le vieillissement. Par exemple, une répondante envisage le vieillissement à l'image du corps de sa grand-mère et du corps de sa mère. Elle fait référence à certains signes de vieillissement du corps: «le corps ratatiné», «les fesses molles».

Afin d'éviter ces problèmes de poids et de corps qui «ramollit», les femmes rencontrées soulignent qu'il est important pour les personnes vieillissantes de se garder en forme. Santé et bonne forme d'une personne s'inscrivent sur le corps, peuvent être «lues» 
"Une forte morale $d u$ devoir et sens de l'effort ressortent clairement des énoncés des femmes rencontrées dans l'articulation apparencesanté.» par les autres. Pour une répondante par exemple, «tu peux distinguer un corps en forme même s'il y a un certain âge». Une autre femme décrit le vieillissement comme ce que chaque individu en fait. Elle dit qu'elle est fière de son apparence alors que certaines personnes «ont toujours mille excuses» pour ne pas prendre soin de leur apparence. Cette informatrice souligne que chacune doit faire les efforts nécessaires afin de bien paraitre même si elle vieillit. Ce sens du devoir est lié à la fierté de la production d'une belle apparence. Pour elle, sens du devoir et fierté vont de pair. L'idée de bien paraître suppose l'entretien corporel (PagèsDelon 1989). Il s'agit de "prendre soin de son corps», une notion très proche de l'hygiène et de la propreté, telles que définies couramment dans la société occidentale contemporaine. Une forte morale du devoir et sens de l'effort ressortent clairement des énoncés des femmes rencontrées dans l'articulation apparencesanté. En fin de compte, les femmes se disent responsables de leur apparence et de leurs motivations à s'engager dans les pratiques nécessaires à modeler ce corps. Selon les femmes rencontrées, la fierté dans la présentation de soi est nécessaire pour un entretien corporel approprié. Cette fierté peut aussi refléter «l'absence de complexe».

Sentiments négatifs et positifs par rapport au corps peuvent coexister chez une même femme. Une répondante dit qu'elle n'a pas de complexes tout en étant insatisfaite de son poids. Une autre informatrice se dit gênée par son corps nu alors qu'elle est fière de son apparence vestimentaire («c'est pas le corps dans le sens, quand le corps est habillé, je suis pas gênée») et du soin qu'elle met à sa présentation d'elle-même. Ainsi les vêtements et le corps nu peuvent refléter deux logiques ou réalités entièrement différentes chez la même personne.

Les entretiens montrent donc les sentiments contradictoires que vivent les femmes par rapport au corps et aux apparences. Ils montrent que des logiques différentes coexistent chez la même personne. Ces contradictions peuvent être interprétées comme des signes d'aliénation fondamentale des femmes. Pour ma part, je propose que les entretiens révèlent à la fois une grande lucidité chez les femmes rencontrées et la dificulté d'aller à l'encontre des 
normes sociales. Les femmes rencontrées sont très conscientes des obligations corporelles qui pèsent sur elles en tant que femmes. Qu'en est-il du style? Et particulièrement du visage, cet élément crucial du corps pour la production et la reconnaissance de l'identité individuelle? La prochaine section examine comment les dimensions apparence-santé-vieillissement s'articulent autour de la notion de «look naturel».

\section{Un look «naturel»: 1'envers du «masque»}

Parmi les femmes rencontrées, les femmes les plus âgées en particulier ont souligné l'importance pour elles d'adopter un look «simple» et «naturel» (certaines femmes ajoutent aussi un look «sportif»). Elles ont beaucoup parlé du visage et du maquillage. Pour décrire l'allure «naturelle», elles ont fait référence à la notion de «masque» ou à un «look» à éviter. Cette distinction entre le «naturel» et le «masque» est fondamentale dans les énoncés des femmes. Mais étant donné que la notion de «masque» est toujours invoquée pour définir son opposé, le «naturel», ces deux notions sont indissociables.

Comment les femmes décrivent-elles le «masque»? Par quelles pratiques d'apparence? Le maquillage est souvent défini comme «masque» lorsqu'il est jugé «excessif». Le maquillage peut aussi être décrit comme «masque» si on le considère «mal fait». Excès et incompétence se recoupent dans les énoncés des femmes et dans leurs regards sur elles-mêmes et sur d'autres femmes. Une répondante de 70 ans dit qu'elle veut «bien» se maquiller. Si elle «s'habitue» au look des autres femmes beaucoup plus maquillées qu'elle ("y en a qui ont l'air de masques... d'un paquet de pâte dans la tête»), elle veut néanmoins éviter de faire cette erreur pour elle-même.

Plusieurs répondantes soulignent qu'elles désirent éviter l'excès («ne pas exagérer») et mettent l'accent sur l'importance de l'hygiène et de la propreté ("bien nettoyer la peau»). Mais si elles ne veulent pas porter de «masque», elles ajoutent qu'elles ne veulent pas non plus avoir l'air «négligé». Présenter un look «naturel» ne veut pas dire se laisser aller. Ce look requiert une intervention minimale sur le corps. En fait, cette conception de maquillage est 
"Une répondante dit que l'apparence, «c'est le reflet de ce que tu es dans le fond". L'apparence ne doit pas servir à cacher la personne, ce qu'elle vit, qui elle est.»

"C'est donc particulièrement en terme de catégorie d'âge que les membres de notre société évaluent les pratiques d'apparence et le degré de maquillage acceptable ou inacceptable.» très près des notions de propreté et d'hygiène. Finalement c'est un maquillage non théâtral, un maquillage qui ne s'avoue pas comme tel que les femmes recherchent. Paradoxalement, pour produire un look «naturel» l'apparence doit être soignée.

De plus l'apparence doit refléter l'identité et les humeurs de la personne. Une répondante dit que l'apparence, «c'est le reflet de ce que tu es dans le fond». L'apparence ne doit pas servir à cacher la personne, ce qu'elle vit, qui elle est. Les répondantes partagent cette morale de la transparence, cette idée que les apparences doivent exprimer honnêtement l'identité individuelle (ou le soi). Dans cette morale, les "artifices» sont répréhensibles. ${ }^{8}$

Pour les femmes âgées, une autre signification d'un maquillage «inapproprié» s'ajoute aux connotations d'incompétence et d'artifices : il s'agit du ridicule dont elles font souvent les frais. Avec le vieillissement, la peur de «l'excès» ressort de façon particulièrement marquée du discours des femmes, notamment en ce qui a trait au maquillage et au parfum. Pour les femmes plus âgées, le maquillage doit être plus «discret» que pour les plus jeunes femmes. Les femmes plus âgées disent aussi préférer des vêtements discrets, «sportifs» ou plus «classiques». Les notions de naturel et de discrétion sont donc aussi invoquées pour les choix des vêtements.

C'est donc particulièrement en terme de catégorie d'âge que les membres de notre société évaluent les pratiques d'apparence et le degré de maquillage acceptable ou inacceptable. Simultanément, les degrés de maquillage marquent et constitutent les catégories d'âge. Pour les femmes les plus âgées, le risque du ridicule est grand. Leur savoir-faire doit donc reconnaitre les limites posées par les normes sociales quant aux catégories d'âge et leurs significations pour les femmes. Il n'est donc pas surprenant que plusieurs femmes disent préférer un «look naturel».

\section{La désexualisation du vieillissement chez les femmes: cantonnement ou libération?}

$\mathrm{Si}$, avec le vieillissement, la santé devient une préoccupation de plus en plus saillante dans les pratiques corporelles des femmes, l'apparence demeure donc importante, tout en changeant de 
forme. Santé, beauté et apparence plus généralement prennent des configurations particulières dans la production sociale de la vieillesse. Dans la société occidentale contemporaine, les individus doivent pouvoir produire et «lire» sur le corps de soi et des autres la bonne forme, la bonne santé. Quelles sont les conséquences de ces obligations sociales pour les femmes plus âgées?

D'une part, les pressions pour que les femmes plus âgées restent jeunes sont réelles. Chez les classes moyennes et supérieures par exemple, le recours à la chirurgie esthétique offre la possibilité de repousser l'apparence du vieillissement. Cette possibilité se traduit en pressions pour les femmes de se faire remonter le visage («face lift») et d'avoir recours à d'autres procédures. Certaines femmes éprouvent de la colère face à l'obligation sociale qu'ont les femmes de toujours tenter de garder une apparence jeune. D'autres disent qu'elles ne s'inquiètent pas encore à ce sujet, même si c'est un sujet de conversation entre femmes dans la quarantaine. D'autre part, les femmes vieillissantes doivent éviter d'enfreindre certains paramètres. Elles doivent éviter par exemple d'avoir l'air «trop

«En ce sens, il semble qu'on ne peut échapper aux discours normatifs de la féminité qui mettent l'accent sur la jeunesse dans l'apparence des femmes. Cependant, les femmes âgées doivent donc trouver un délicat équilibre en évitant de paraitre vieilles tout en ayant l'air «de leur âge».» jeune pour leur âge». En ce sens, il semble qu'on ne peut échapper aux discours normatifs de la féminité qui mettent l'accent sur la jeunesse dans l'apparence des femmes. Cependant, les femmes âgées doivent donc trouver un délicat équilibre en évitant de paraitre vieilles tout en ayant l'air «de leur âge».

Paradoxalement, le vieillissement peut simultanément apporter une certaine désexualisation ou libération de la séduction pour les femmes. La sexualité des femmes d'un certain âge ne semble plus possible ni intéressante selon la construction sociale actuelle de la vieillesse dans la société occidentale contemporaine. La séduction (selon des normes hétérosexistes) n'est plus socialement obligatoire. Cet autre aspect du vieillissement peut être bienvenu pour les femmes qui ont eu une vie très difficile. Par exemple, les femmes qui ont été abusées et battues par leur mari pendant de nombreuses années et qui se sont sorties de la relation, vivent le vieillissement comme une libération. Une de ces femmes souligne en riant qu'elle est contente de vieillir et qu'elle était heureuse lorsqu'elle a eu quarante ans car elle s'était libérée de son mari.Vieillir lui fait peur à cause de sa condition physique, car elle a de graves 
problèmes de santé. Elle ajoute: «mon âge ne me fait pas peur, ma condition physique me fait peur». Malgré ses problèmes de dos et sa situation financière difficile, elle ne peut plus vivre autant de haine pour son corps que quand elle vivait avec son mari. ${ }^{9}$

\section{L'ethnicisation du corps et la difficulté d'être minoritaire}

Jusqu'ici ce texte suggère que les femmes francophones en Ontario vivent leurs corps de façon similaire à l'ensemble des femmes nord-américaines et plus largement des femmes de la société occidentale. Est-ce qu'en plus de partager ce rapport aux discours et pratiques féminines dominantes, les femmes francophones en Ontario vivent leurs corps de façon bien à elles ? Dans cette section, je pose quelques questions sur l'ethnicisation du corps des femmes francophones minoritaires. Est-ce que les femmes francophones en Ontario produisent leurs corps comme signes distinctifs de la majorité anglo-saxonne et, si oui, comment? Y at-il une difficulté de vivre en tant que femme francophone minoritaire qui s'articule précisément autour de leur corporéité?

«Dans les entretiens, les femmes rencontrées ne réferent pas toutes explicitement à l'ethnicisation du corps en tant que francophones minoritaires. Cependant, quelques thèmes émergent qui suggèrent des pistes de recherche sur les pratiques et les représentations sociales de l'ethnicisation $d u$ corps.»
Dans les entretiens, les femmes rencontrées ne réferent pas toutes explicitement à l'ethnicisation du corps en tant que francophones minoritaires. Cependant, quelques thèmes émergent qui suggèrent des pistes de recherche sur les pratiques et les représentations sociales de l'ethnicisation du corps. Dans les pages qui suivent, je m'attarde à quelques valeurs et pratiques importantes pour les femmes rencontrées. Ces dimensions de la vie des femmes s'articulent à plusieurs axes de différenciation sociale qui s'entrecroisent et sous-tendent les énoncés des femmes sur le corps. Ces axes sont bien sûr le genre ou sexe social, mais aussi l'âge, les classes sociales, les professions et occupations, l'appartenance «raciale» et ethnique, les régions d'habitation et trajectoires de vie, etc. Cette section examine particulièrement les thèmes suivants: la "différence» et la fierté canadienne française; la modestie et l'influence de la religion catholique; entre québécoises et anglophones de l'Ontario, la difficulté d'être minoritaire et finalement, le corps et la minorisation des femmes. 


\section{«Différence» et fierté canadienne-française}

Certaines femmes ne voient pas de différences entre l'apparence des francophones et l'apparence des anglophones en Ontario. D'autres suggèrent que la distinction anglophone/ francophone n'en dit pas assez sur le rapport entre ethnicité et apparence. Par exemple, une répondante de 35 ans qui a vécu dans nombre de provinces canadiennes et à l'étranger remarque :

Je suppose qu'il y a quand même certaines, certains stéréotypes. Dans les deux sens.(...) Et encore... on vit dans une société tellement métropolitaine... À Toronto... même les femmes francophones sont d'origines tellement différentes; c'est peut-être différent pour quelqu'un qui va à Montréal, c'est plus homogène si tu regardes les femmes francophones puisque t'as la majorité des femmes qui sont francophones, (...) mais à Toronto, une femme francophone a autant de chances de venir d'Algérie ou de Tunisie, d'être juive ou arabe.

«Dans la région de Toronto, le pluralisme ethnique et racial s'inscrit sur les corps selon de multiples possibilités. La dichotomie anglophone/ francophone uniquement de race blanche n'est pas pertinente dans ce contexte.»
Dans la région de Toronto, le pluralisme ethnique et racial s'inscrit sur les corps selon de multiples possibilités. La dichotomie anglophone/ francophone uniquement de race blanche n'est pas pertinente dans ce contexte. ${ }^{10} \mathrm{La}$ dimension régionale elle-même est cruciale dans les distinctions que font les femmes au sujet de la minorisation. Le Québec et plus particulièrement la région de Montréal est une référence très présente dans les énoncés des femmes. Celles qui ont vécu dans plus d'une région ont tendance à comparer ces régions entre elles. Par exemple, les femmes qui vivent à Sudbury mais qui ne viennent pas de là ou qui y sont depuis peu de temps comparent Sudbury avec d'autres villes en termes d'apparences ou de «looks» (Sudbury par rapport à Ottawa et autres permutations). À l'intérieur d'une même région, d'autres distinctions émergent. Rhéa Pointe (75 ans, Sudbury) par exemple, distingue les paroisses entre elles. Elle donne l'exemple d'une paroisse plus ancienne ou les gens, selon elle, sont aussi plus vieux et mieux habillés, portent des chapeaux, par exemple. Pour elle, l'aspect générationnel est donc particulièrement important et lié 
"Différences régionales et différences de style se recoupent et se reflètent dans les énoncés des femmes rencontrées." à une histoire spécifique. Les femmes qui vivent à Ottawa tendent aussi à faire des comparaisons avec Hull et Gatineau. Ottawa est une ville frontière et en ce sens, les références immédiates des femmes se font souvent à partir de la grande région d'OttawaHull. Par exemple une répondante de quinze ans dit en riant que ses amies avaient, il y a deux ans, l'habitude de l'appeler «Gatineau Power», "comme les filles de Gatineau, il paraît qui sont ben chromées tsé, les gros cheveux crêpés, le gros rouge à lèvres». Maintenant elle dit qu'elle «essaie de garder ça assez simple».

Différences régionales et différences de style se recoupent et se reflètent dans les énoncés des femmes rencontrées. Les différences de goût (la définition du beau et du laid, du simple et de l'excessif) sont liées aux différences de classes sociales qui soutendent ces énoncés — classe moyenne supérieure versus classe ouvrière. Une professionnelle d'Ottawa (32 ans) se dit surprise du look des francophones de l'Outaouais car selon elle, ces francophones ont pour la plupart un bon revenu. À salaire égal, elle trouve qu'elles ont moins de goût que les Québécoises de Montréal et Québec.

Cette répondante refute l'idée de la classe sociale comme facteur explicatif de différence. Mais d'autre part, dans le même entretien, elle distingue entre l'apparence du personnel de soutien de l'apparence des professionnelles francophones, les premières étant généralement des francophones de l'Outaouais. C'est d'ailleurs souvent au bureau que les professionnelles observent ces différences de façon non équivoque: il y a peu de femmes francophones parmi les professionnelles et généralement elles viennent de Montréal et Québec. Elles se démarquent clairement des anglophones professionnelles. Selon elle, ces dernières s'habillent de façon "plus conservatrice»:

Ça va être vraiment le petit tailleur là, mais très très straight qu'elles vont pouvoir mettre, même si elles ont trente ans maintenant, à 50 ans elles vont pouvoir le mettre encore et elles seront pas déphasées. Elles vont plus aller dans les petites jupes droites, tu sais petit tailleur, style Chanel là ou double breast bien simple. 
Une autre répondante ajoute que les francophones sont plus à la page et s'habillent de façon «plus jeune». Selon elle, la différence entre professionnelles anglophones et francophones est une question de valeurs. Les anglophones semblent «moins intéressées» à l'apparence même lorsqu'elles n'ont pas de contraintes financières. Parmi les femmes rencontrées, les professionnelles en particulier ont souligné que les francophones se distinguent clairement des anglophones par leur bon goût et leur créativité. Rolande Tremblay, pour sa part, trouve que la distinction entre

"Celles qui perçoivent une fierté et un sens de supériorité esthétique pour l'ensemble des "canadiennesfrançaises» soulignent que cette supériorité esthétique se manifeste non seulement par de la fantaisie et la créativité dans les vêtements mais aussi dans l'ensemble de la vie quotidienne. » francophones et anglophones est pertinente partout, dans tous les milieux. Elle souligne que l'apparence reflète une "fierté d'être canadienne française». Quelques femmes seulement ont suggéré que les anglophones paraissent mieux que les francophones. Celles qui perçoivent une fierté et un sens de supériorité esthétique pour l'ensemble des «canadiennes françaises» soulignent que cette supériorité esthétique se manifeste non seulement par de la fantaisie et la créativité dans les vêtements mais aussi dans l'ensemble de la vie quotidienne. Une répondante de 41 ans, par exemple, dit que les anglophones sont plus utilitaires et éprouvent moins de plaisir tactile à manger; les francophones, selon elle, sont plus latines et ont "plus de zest, de feeling" pour tous les aspects de la vie.

\section{Modestie et influence de la religion catholique}

«La religion catholique a-t-elle eu une influence marquée sur les pratiques corporelles et d'apparences des «canadiennesfrançaises»?»
Si les francophones ont des influences latines, elles ont aussi inextricablement subi des influences de la religion. Comment ces dimensions s'articulent-elles pour les francophones minoritaires en Ontario dans le rapport au corps? La religion catholique a-t-elle eu une influence marquée sur les pratiques corporelles et d'apparences des «canadiennes françaises»? La modestie dans l'apparence peut s'expliquer par des racines religieuses. Certaines femmes affirment que la religion catholique a joué un rôle majeur dans la négation du corps. 
Chez mes parents, avec mes parents, on parlait pas beaucoup d'apparence, puis du corps, tu vois; on est catholiques alors, tout ça, c'était tabou... (...) Puis, ma mère était, elle l'est encore là, plutôt à tendances féministes alors, elle essayait de pas trop nous montrer ces côtés là; elle a plutôt poussé, si tu veux, le côté intellect. (...) Alors on n'a jamais, d'une certaine façon... ils nous ont donné une très bonne estime de soi, mais sur un plan seulement.

Selon la répondante, la religion catholique et le féminisme ont le même effet: le corps est tabou, le plaisir et l'intérêt pour le corps sont défendus. De plus, la dichotomie esprit ou l'intellectsupérieur/ corps-dénigré est renforcée. Cette répondante, qui se définit pourtant comme féministe, ne pratique plus la religion catholique. Elle dit vouloir apprendre à se réapproprier son corps, sans culpabilité.

Mais pour certaines femmes, la modestie des apparences n'est pas attribuable à la religion en tant que telle. Madame Desroches, qui est âgée de 75 ans, par exemple, dit que sa mère était «très sévère» et que dans sa famille, durant son enfance et adolescence, il «ne fallait surtout pas se trouver jolie», mais il s'agissait d'avoir l'air «propre». J'ai demandé à cette répondante si l'attitude de sa mère était liée à la religion. Elle répond que non, «la religion défendait pas aux femmes d'être jolies»! Elle ajoute que sa mère et sa grand-mère avaient beaucoup souffert. Sa grand-mère avait perdu onze enfants en quinze ans. Selon cette répondante, qui

"Cette question demeure cependant une piste de recherche intéressante à poursuivre dans l'analyse de la vie quotidienne des femmes francophones nées en Ontario.» pratique encore la religion catholique, le malheur rend les gens «rigides» et "pessimistes». Ainsi, pour certaines répondantes, les traces de la religion catholique ne sont pas faciles à distinguer d'autres caractéristiques de la francophonie minoritaire en Ontario dans le rapport au corps. Cette question demeure cependant une piste de recherche intéressante à poursuivre dans l'analyse de la vie quotidienne des femmes francophones nées en Ontario. 


\section{Entre Québécoises et anglophones de l'Ontario : la difficulté d'être francophone minoritaire}

"Quelques femmes décrivent explicitement leurs pratiques d'apparence comme signifiant une position sociale «d'entre deux».»
Pour plusieurs femmes, le Québec est une référence cruciale et en particulier le look des femmes montréalaises. Les femmes rencontrées insistent sur leur style unique. Certaines expliquent qu'elles adoptent (ou gardent) des pratiques similaires aux Montréalaises, tout en empruntant au look des anglophones en Ontario. Dominique, par exemple, vient de Montréal. Quand elle est arrivée à Ottawa, elle trouvait «effrayant» de voir «les femmes en tailleurs dans la rue avec leurs petits bas puis leurs espadrilles». Maintenant elle dit en riant porter des espadrilles pour aller au bureau. Mais elle spécificie que ce sont «des petites espadrilles fleuries là, c'est pas des grosses Adidas Nike là».Avec cette derrnière remarque, la répondante indique qu'elle a tout de même du "goût» et n'a pas exactement l'air d'une anglophone.

Quelques femmes décrivent explicitement leurs pratiques d'apparence comme signifiant une position sociale «d'entre deux». Par exemple, Blanche Beaudry se dit influencée par les Québécoises et par les anglophones en Ontario, sans appartenir pour autant à l'une ou à l'autre des catégories. Pour elle, le Québec demeure le modèle à atteindre et elle préfere y acheter ses vêtements. Mais elle n'a pas encore atteint ce modèle, elle dit avoir «des influences anglaises trop marquées». Madame Beaudry ajoute que sa belle-famille (québécoise) l'appelle «l'anglaise», alors qu'elle est Franco-Ontarienne. Elle porte «des costumes sobres, des couleurs plus sobres, des bijoux moins voyants, une coupe de cheveux moins dernier cri» que les Québécoises. Selon elle, «les Franco-Ontariennes là-dedans, ont ni l'avantage de l'un ni de l'une parce que je trouve qui sont pas aussi bien mises que les Québécoises». Pour cette répondante, cette situation «entre les deux» est difficile à vivre. Madame Beaudry est une des seules femmes rencontrées qui lie explicitement cette position «d'entre deux» à la minorisation des femmes francophones en Ontario.Dans 
sa référence explicite à la "différence», elle fait allusion au désavantage d'être minoritaire :

Moi je pense par exemple que la question d'être minoritaire(...) le fait d'être Franco-Ontarien, ça change quelque chose à la perception de ton corps. Je pense que le fait d'être Franco-Ontarien, la difficulté d'être Franco-Ontarien, c'est que t'as pas de masse critique nulle part. T'as pas suffisamment de personnes qui sont comme toi. Donc, que tu sois bourgeois ou que tu sois un ouvrier, que tu sois une ménagère, y en a comme toi mais, pas suffisamment pour que ça crée un milieu homogène dans lequel tu te retrouves puis tu te sens bien. Je nous trouve probablement doublement insécures. Moi, en tout cas, je dirais ça. Moi, je suis terriblement insécure et je pense, c'est beaucoup d'avoir vécu à l'ombre d'une autre culture, de toujours savoir que t'es un peu différent.

D'autres femmes lient le fait d'être minoritaire francophone à la question des classes sociales. Une répondante de 68 ans raconte que sa mère et sa soeur cousaient et faisaient tout pour que la pauvreté de la famille ne paraisse pas: «Y avait une fierté, de la façon de s'habiller là, ça, l'apparence, c'était bien important». Une autre femme décrit la convergence entre classe sociale et ethnicité lorsqu'elle grandissait. En plus des inégalités économiques, les francophones et les anglophones avaient, selon Madame Desroches, et ont peut-être encore, des types physiques bien différents. Les anglophones étaient "plus grandes, plus minces, plus belles et mieux habillées que les Canadiennes françaises». Ces dernières parlaient des anglophones en termes de "grandes anglaises». Ces anglaises avaient «beaucoup de goût», selon cette répondante. Elle observe que ce modèle de l'élégance anglophone persiste encore aujourd'hui à Ottawa. C'est une élégance sobre, un goût des beaux tissus et «pas de fanfreluches». Elle ajoute en riant que les «anglaises» sont distinctes en ce qu'elles ne «changent pas leur personnalité propre, elles vont le... un mot anglais qui me vient là elles vont enhance it». 
"Au-delà de la religion et des inégalités de classes sociales, certaines trouvent difficile d'expliquer l'ethnicisation du corps des francophones minoritaires. Elles hésitent et plusieurs disent qu'elles veulent éviter les clichés et stéréotypes.»
Au-delà de la religion et des inégalités de classes sociales, certaines trouvent difficile d'expliquer l'ethnicisation du corps des francophones minoritaires. Elles hésitent et plusieurs disent qu'elles veulent éviter les clichés et stéréotypes. Pour les femmes de race blanche, il n'est pas facile de trouver les mots, d'articuler cette ethnicisation par le corps. Même dans le cas des femmes qui la perçoivent, les mots peuvent manquer pour décrire cette différence. Ainsi de nombreuses femmes disent que la différence est «difficile à décrire». Le rapport au corps pour les femmes francophones minoritaires de race blanche demeure donc une question à élucider.

\section{Le corps et la minorisation des femmes}

Finalement, quelques femmes conçoivent la minorisation des femmes francophones en Ontario comme relevant de la minorisation des femmes dans leur ensemble par le corps. Le rapport au standard idéal de beauté véhiculé dans les médias est crucial dans cette minorisation, comme l'exprime Rita:

En fait, moi je crois que d'une certaine façon, la Californie (...) c'est un problème justement, (...) Collectivement, représentant des normes que j'ai profondément rejetées, et que vraiment, plus j'y pense, plus la Californie me revient de moins en moins. (...) C'est quand même un monde de Miss America, ce monde-là. (...) Et c'est un centre d'influence visuel donc ... (...) la bonne femme à Entertainment Tonight. (...) Pour moi, c'est ça la Californie d'abord. C'est pas l'individu, c'est la projection collective.

Pour plusieurs, le rapport au corps se vit dans la culpabilité et la souffrance même si les femmes rencontrées sont bien conscientes que ce sont des normes sociales qui sont à la source de leur insatisfaction. Ainsi, plusieurs femmes disent se sentir inadéquates dans tous les domaines de la vie. Elles lient donc l'insatisfaction 
du corps au reste de la vie quotidienne des femmes. Une répondante réfere premièrement aux normes sociales qui vouent les femmes à l'échec («on n'en fait jamais assez»), puis elle spécifie qu'elle parle d'elle en particulier, qu'elle n'est jamais satisfaite d'elle-même. Une autre affirme: «Y a toujours quelque chose qui cloche, t'sé. On trouve toujours, toujours, toujours à redire sur soi-même». Pour elles, cette dureté envers soi-même est à désapprendre dans tous les domaines de la vie. Certaines femmes croient que le succès professionnel et d'autres aspects positifs de la vie d'une femme peuvent l'aider à apprivoiser son corps. En ce sens, avancer en âge peut être source d'espoir.

\section{Conclusion}

Les stratégies de changement social et d'intervention dans les communautés doivent se faire à partir des complexités du vécu des femmes et de leur ludicité critique face aux multiples obligations corporelles qui façonnent leur identité et leur santé. Ce texte a examiné la représentation de soi de quelques femmes francophones minoritaires en Ontario. De cette recherche sur ces femmes et leur rapport au corps émergent des points communs entre les femmes d'une même culture globale ainsi que des «différences» ou spécificités des minorisées.

Les femmes francophones minoritaires partagent avec d'autres femmes de la société occidentale contemporaine des préoccupations de santé et d'apparence qui s'entremêlent, que ce soit pour la question du poids, de l'exercice ou plus généralement de la «bonne forme». Il importe pour les femmes de «se tenir en forme» et d'éviter de se «laisser aller». Les femmes désirent vivre l'épanouissement dans le vieillissement et atteindre (ou maintenir) une bonne santé globale. Les préoccupations esthétiques font partie de ces processus. Les femmes à qui j'ai parlé sont très conscientes des difficultés que posent les normes corporelles de notre société pour les femmes âgées. Elles vivent des tiraillements et des contradictions par rapport à ces normes. Tout en partageant la 
"La théorisation des spécificités du vécu corporel pour les femmes francophones minoritaires en Ontario n'en est qu'à ses débuts. Ce texte s'est penché sur seulement quelques pistes de recherche.» morale de l'effort et de la responsabilité individuelle, elles peuvent parfois refuser une accommodation complète en élaborant des stratégies de recul ou de négociation envers les obligations du corps. Une répondante, par exemple, dit que finalement les relations avec les autres sont plus importantes que l'image de son corps, que «ça vaut pas la peine» de se tourmenter.

La théorisation des spécificités du vécu corporel pour les femmes francophones minoritaires en Ontario n'en est qu'à ses débuts. Ce texte s'est penché sur seulement quelques pistes de recherche. Les représentations sociales doivent être explorées en incluant mais en allant aussi au-delà des clichés et stéréotypes. Finalement, les chercheures et intervenantes ont tout à gagner en s'attardant aux subtilités des énoncés des femmes sur le rapport au corps et leur situation de minoritaires. Les hésitations des femmes qui voient la «différence» sans pouvoir facilement la décrire apparaissent comme une voie particulièrement fructueuse à poursuivre dans l'étude de l'identité chez les femmes des francophonies minoritaires.

\section{Bibliographie}

BEAUSOLEIL, Natalie (1994). «Makeup in Everyday Life:An Inquiry Into The Practices Of Urban American Women Of Diverse Backgrounds», Many Mirrors: Body Image and Social Relations, s. la dir. de Nicole Sault, New Brunswick (N.J.), Rutgers University Press.

BORDO, Susan (1993). Unbearable Weight: Feminism, Western Culture and the Body, Berkeley, University of California Press.

CAMPBELL, Marie et Ann MANICOM (s. la dir. de) (1995). Knowledge, Experience, and Ruling Relations, Toronto, University of Toronto Press.

CARDINAL, Linda (1994). "Ruptures et fragmentations de l'identité francophone en milieu minoritaire; un bilan critique», Sociologie et Sociétés, vol. XXVI, no1, 71-86.

CHERNIN, Kim (1981). The Obsession: Reflections on the Tyranny of Slenderness, New York, Harper and Row.

CORIN, Ellen E. et al. (1990). Comprendre pour soigner autrement, Montréal, Les Presses de l'Université de Montréal.

DESLAURIERS, J.-P. (1991). La recherche qualitative: guide pratique, Montréal, McGraw -Hill.

DEVAULT, Marjorie (1991). Feeding the Family, Chicago, University of Chicago Press.

DEVAULT, Marjorie (1990). «Talking and Listening from Women’s Standpoint: Feminist Strategies for Interviewing and Analysis», Social Problems,vol. 37, 96-116. 
EMERSON, Robert (s. la dir. de) (1983). Contemporary Field Research, Boston, Little, Brown and Company.

FEATHERSTONE, Mike, Mike HEPWORTH et Bryan S. TURNER (s. la dir. de) (1991). The body: Social Process and Cultural Theory, London, Sage.

FEHER, Michel, Ramona NADAFF et Nadia TAZI (s. la dir. de) (1989). Fragments For A History Of The Human Body, New York, Zone, 3 t.

FISKE, John, (1993). Power Plays Power Works, London, Verso.

GROSZ, Elizabeth (1992). «Le corps et les connaissances: le féminisme et la crise de la raison», Sociologie et Sociétés, vol. 24, no 1, 47-66.

GROSZ, Elizabeth (1994). Volatile Bodies:Toward A Corporeal Feminism, Indianapolis, Indiana University Press.

HUBERMAN,A.M. et M.B.MILES (1991). Analyse des données qualitatives: recueil de nouvelles méthodes, Bruxelles, Université De Boeck. Trad. de l'anglais par C. De Backer et V. Lamongie.

KING, Dixie L. (1994). «Food, Sex, and Salvation: The Role of Discourse in a Recovery Program ofr Eating Disorders", Many Mirrors: Body Image And Social Relations, s. la dir. de N. Sault, New Brunswick ( N.J.), Rutgers.

LADOUCEUR, Lise (1996). «Différence chez les personnes âgées: mise en contexte », Reflets, vol. 2, no 2, 82-97.

MASCIA-LEES, F rances E. et Patricia SHARPE (s. la dir. de) (1992). Tattoo, Torture, Mutilation And Adornment:The Denaturalization OfThe Body In Culture And Text, Albany, State University of New York Press.

MILLMAN, Marcia (1980). Such a Pretty Face, New York, Berkeley Books.

MISHLER, Elliot G. (1986). Research Interviewing: Context And Narrative, Cambridge (M.A.), Harvard University Press.

PAGÈS-DELON, Michèle. 1989. Le corps et ses apparences: l'envers du look. Paris, l'Harmattan. Logiques sociales.

ROBERTS, Helen (s. la dir. de) (1981). Doing Feminist Research, London, Routledge \& Kegan Paul. SMITH, Dorothy E. (1987). The Everyday World As Problematic:A Feminist Sociology, Boston, Northeastern University Press.

SYNNOTT, Anthony (1993). The Body Social, New York, Routledge.

SZEKELEY, E. (1988). Never Too Thin, Toronto, The Women's Press.

TURNER, Bryan S. (1984). The Body \& Society, Oxford, Basil Blackwell.

\section{Notes}

1. Je remercie vivement Michèle Kérisit, Gertrude Mianda, Caroline Andrew et deux évaluatrices anonymes de leurs commentaires et suggestions.

2. Le corps et l'apparence constituent des dimensions importantes de l'identité, liées à des conditions matérielles concrètes d'existence (Beausoleil 1994; Synnott 1993). De plus, le corps est 
non seulement un lieu important de l'élaboration de l'identité individuelle mais le corps et les apparences sont fondamentalement sites de l'inscription de la culture (Featherstone et al 1991; Feher et al 1989a,b.c; Fiske 1993; Grosz 1992; Grosz 1994; Mascia-Lees 1992; Synnott 1993; Turner 1984).

3. Ces entretiens se sont déroulés sous le mode de la conversation, avec ma participation active. Je ne pouvais pas être distante des femmes rencontrées. Quelques femmes m'ont d'ailleurs demandé comment je vis mon corps (d'autres si j'ai des enfants, etc.). Certaines ont commenté sur mon apparence: par exemple, une répondante dit que j'étais habillée «comme une maitresse d'école» (jupe courte noire, collants noirs, gilet noir et veston en tweed). D'autre part, quelques femmes m'ont demandé comment d'autres femmes interviewées vivaient leur apparence. J'étais perçue comme une médiatrice ou un lien entre les femmes.

4. Le vécu corporel et les apparences sont à la fois des sujets courants de conversation entre des femmes qui se connaissent et des femmes qui ne se connaissent guère. Dans une étude précédente j'ai montré comment une pratique d'apparence, le maquillage, fait partie d'un "monde de femmes" ou "culture de femmes" (Beausoleil 1994).

5. J'ai rencontré des femmes à Toronto (11), à Ottawa (12), et à Sudbury (7). Les entretiens ont été effectués entre mai et décembre 1996. Pour trouver/recruter les interviewées, j’ai utilisé la méthode dite «boule de neige.» J'ai distribué un formulaire de recrutement à des connaissances (femmes francophones en Ontario) et amies. J'ai aussi affiché ce formulaire dans des centres de conditionnement physique et autres endroits publics.

6. Dans cet article, les entretiens utilisés ont été réalisés avec des femmes de race blanche. Cela s'explique par le fait que les personnes d'autres groupes raciaux que j'ai rencontrées pour la recherche en Ontario sont dans l'ensemble plus jeunes que la quarantaine et ne m'ont pas parlé du processus de vieillissement.

7. La plupart des femmes se trouvent «trop grosses», quel que soit leur poids ou leurs proportions. Dans ce contexte social, 
de très nombreuses femmes vivent leur corps avec beaucoup de douleur. La minceur/grosseur est une préoccupation de presque tous les instants et est aussi un sujet de discussion courant entre les femmes. Étant donné l'obligation de minceur, le rapport des femmes à la nourriture est particulièrement tourmenté. Les femmes ont eu à se priver tout en ayant la responsabilité de nourrir leur famille ou d'autres personnes dans des occupations de service (DeVault 1991).

8. Cette correspondance entre apparence et identité est un thème fondamental des discours sur l'apparence dans la culture occidentale contemporaine. L'axe nature/masque, très chargé émotivement, est constamment invoqué dans les liens que font les femmes entre apparence et identité (Beausoleil 1992). L'apparence se doit de réfléter et même «d'exprimer» la psyché de chaque individu. Mais il y a toujours tension entre l'apparence comme reflet du soi (ou de l'âme) et l'apparence comme masque de la «vraie» personnalité (Beausoleil 1994; Peiss 1990; Synnott 1993). Les entretiens que j’ai effectués confirment cette tension.

9. La situation des femmes plus âgées par rapport à l'emploi peut aussi permettre une certaine flexibilité dans le domaine des apparences.Ainsi les femmes à la retraite n'ont plus à répondre aux obligations, aux codes explicites et implicites des apparences, du marché du travail. En ce sens, la retraite peut être une sorte de libération. Ma recherche précédente a montré l'importance de l'emploi dans les décisions des femmes quant à l'apparence (Beausoleil 1992).

10.Je prépare actuellement un article sur le pluralisme et l'ethnicisation du corps chez les femmes francophones en Ontario. 